\title{
MIMO TRANSMIT STRUCTURE ASSIGNMENT WITH QOS PROVISION IN WIRELESS SYSTEMS
}

\author{
W. C. Freitas Jr. \& F. R. P. Cavalcanti \\ Wireless Telecom Research Group (GTEL) \\ Federal University of Ceará (UFC) \\ Fortaleza-CE, Brazil
}

\section{ABSTRACT}

In this paper, we propose a suboptimal algorithm to assign MIMO transmit structures subject a required quality of service (QoS). In particular, we use the error probability of MIMO structures designed to achieve diversity and multiplexing gains as the QoS target. This suboptimal algorithm provides two MIMO structures that are hybrids between diversity and multiplexing.

\section{INTRODUCTION}

The market of wireless communications continues to grow. The next generation (XG) of the wireless communication systems will provide a wide variety of services, ranging from high quality voice communication to wireless multimedia services, to anyone, in anyplace in anytime, following the always best connected (ABC) concept in a multi radio access network (RAN). Each service has different quality of service (QoS) requirements, motivating the pursuit for high data rates in the air interface with higher spectral efficiency.

One way to achieve the high data rates required by the XG wireless systems is the use of multiple antennas in both transmitter and receiver, creating a multiple-input multiple-output (MIMO) channel. Using multiple antennas, under certain conditions the channel capacity increases almost linearly with the smaller number of antennas [1]. This capacity increase is known as spatial multiplexing gain and it enables the high data rates required by XG systems. Besides high data rates, with multiple antennas it is also possible to achieve diversity gain using the spatial dimension created by the multiple antennas to send redundancy, as in traditional channel coding approaches.

In [2], Zheng \& Tse derive a fundamental tradeoff between the spatial gains in a MIMO channel: multiplexing and diversity. Basically, in [2] it is shown that increasing the multiplexing gain the diversity gain is necessarily decreased, and vice-versa. In other words, schemes designed to exploit spatial multiplexing can not capture the maximal transmit diversity gains and, on the other hand, schemes designed to take advantage of the diversity must have a reduced multiplexing gain.

Regarding the tradeoff between spatial multiplexing and diversity gains present in MIMO channels, we can formulate a resource allocation problem, assigning either diversity or multiplexing in accordance of the MIMO channel quality and QoS requirements using the error probability analysis presented in [3]. Since optimal solutions to such resource allocation problems are computationally complex, in this

\author{
R. R. Lopes \\ State University of Campinas (Unicamp) \\ Communications Department (DECOM) \\ Campinas-SP, Brazil
}

paper we propose a low-complexity suboptimal solution. Our proposal starts assigning orthogonal space-time block codes (OSTBC) to fulfill the QoS requirement and multiplexing layers are assigned if the QoS target is achieved with the first assigning, thus, obtaining a higher data rate. Instead, other OSTBC layer is allocated to provide more diversity.

This paper is organized as follows. In Section II, we describe the MIMO channel model and the MIMO transmit structures considered in this paper. Section III presents the MIMO tradeoff resource allocation as an optimization problem. In Section IV we propose a MIMO transmit structure assignment that is a suboptimal algorithm that respects a QoS requirement, using an error probability analysis proposed in [3]. In Section $\mathrm{V}$, we present simulation results of our proposal and then we conclude the paper.

\section{MimO Channel Model And TRAnsmit STRUCTURES}

In this section, we describe the MIMO channel model regarded in this paper and review some strategies that achieve diversity and multiplexing gains in a MIMO channel.

\section{A. MIMO Channel Model}

In this paper, we consider a transmitter equipped with an $M$-element antenna array and a receiver equipped with an $N$-element antenna array. This system, referred to as (MTx-NRx), is depicted in Figure. 1. The transmitted signals are assumed to go through a random channel matrix $\mathbf{H}$. The wireless channel is assumed to have rich scattering and flat fading. The fading between each transmit and receive antenna pair is assumed to be independent and the entries of $\mathbf{H}$ are circularly symmetric Gaussian random variables. The quasi-static block fading model is assumed. Furthermore, we assume i.i.d circularly symmetric Gaussian noise samples. For all the MIMO transmission structures, we assume that the total transmit power is fixed (normalized to 1) and equally divided across the transmit antennas. Ideal symbol timing and pulse shaping are assumed at the transmitter and receiver. Thus, we can relate the transmit and receive symbols in $T$ consecutive symbol periods complex baseband form and at the symbol rate

$$
\mathbf{X}=\sqrt{\frac{\rho}{M}} \mathbf{H S}+\mathbf{V}
$$

where $\mathbf{X}=\left[\begin{array}{llll}\mathbf{x}_{1} & \mathbf{x}_{2} & \cdots & \mathbf{x}_{T}\end{array}\right] \in \mathcal{C}^{N \times T}$ denotes the matrix of complex received symbols during $T$ consecutive symbol periods, $\mathbf{S} \in \mathcal{C}^{M \times T}$ denotes the matrix of the complex transmitted symbols having dimension $M \times T, \mathbf{H} \in \mathcal{C}^{N \times M}$ 


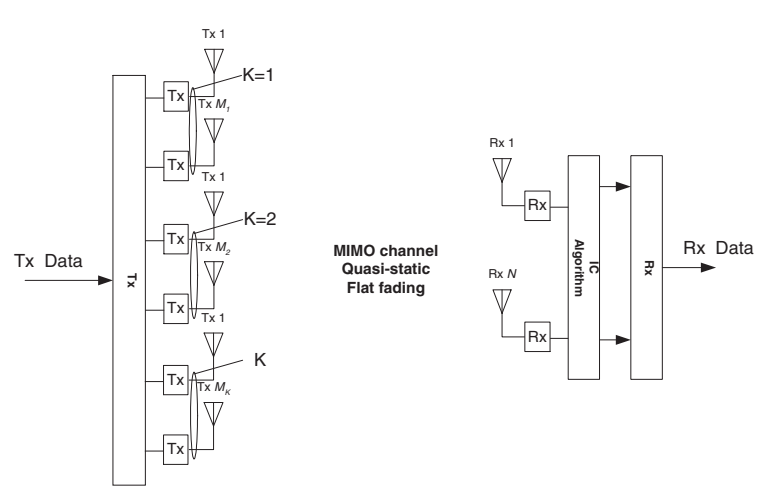

Figure 1: Generic MIMO transmit structure.

denotes the channel matrix, $\mathbf{V} \in \mathcal{C}^{N \times T}$ is the zero-mean, unit variance and complex-Gaussian distributed noise that is spatially and temporally white, and $\rho$ is the signal-to-noise ratio (SNR).

\section{B. MIMO Transmit Structures}

Structures that provide diversity and multiplexing gains have been studied for almost a decade. In [2], Zheng \& Tse shown, using the information theory approach, that the maximization of one possible spatial gain decreases the other one, and vice-versa. In other words, structures that provide diversity gain, e.g., OSTBC, can not achieve the maximal possible spatial multiplexing gain. Indeed, often OSTBC provide diversity gain with a loss in the data rate. On the other hand, structures focused on multiplexing, e.g., the vertical Bell layered space-time (VBLAST), can achieve the maximal multiplexing gain, but can just capture diversity gain in the receiver.

Since, OSTBC schemes achieve MIMO diversity decreasing the capacity and VBLAST structures achieve the maximal channel capacity with lower diversity than maximal, we can formulate an optimization problem in which the MIMO transmit structure is assigned in accordance with the MIMO channel and QoS requirement of the service, through an error probability target. Following, we formulate the optimization problem.

\section{OPtimizATION PROBLEM Formulation}

Let a MIMO system composed by $M$ transmit and $N$ receiver antennas. In this system the $M$ transmit antennas can be divided in $K$ layers, such that $M=\sum_{k=1}^{K} M_{k}$, see Figure 1. A layer may consist of the stream of symbols at the output of a OSTBC, which is sent to a group of antennas, or of an uncoded stream, which is transmitted from a single antenna. Without loss of generality, we will define the data rate per channel use (pcu) of MIMO transmit structures as

$$
R=\frac{\text { number of transmitted symbols }(S)}{\text { number of symbol periods }(T) \text { to transmit } S \text { symbols }} .
$$

Let $R_{k}$ be a data rate of a particular layer $k$.

The objective of the optimization problem thought to the MIMO case is maximize the whole data rate of the MIMO system subject to a constraint in the error probability in such way that the QoS requirement must be fulfilled with total power transmission limitation. We can formulate such problem as

$$
\begin{aligned}
& \max _{K, R_{k}} \sum_{k=1}^{K} R_{k} \\
& \text { subject to } \\
& \operatorname{Pr}(\text { error } \mid \mathbf{H}) \geq \operatorname{Pr}(\text { error } \mid \mathbf{H})_{\text {target }} \\
& \quad \sum_{k=1}^{K} P_{k} \leq P_{T},
\end{aligned}
$$

where $\operatorname{Pr}($ error $\mid \mathbf{H})$ is the error probability of the MIMO system and $\operatorname{Pr}(\text { error } \mid \mathbf{H})_{\text {target }}$ is the error probability target that must be respected to fulfil the QoS required by some wireless service, e.g. packet data transmission. The maximal total power transmission possible is $P_{T}$, thus, $\sum_{k=1}^{K} P_{k} \leq P_{T}$.

Such optimization problems are generally very hard to solve optimally and due to complexity usually result in a significant computational overhead. One solution to the complexity issue is the relaxing of one or more constraints of the optimization problem which can be solved efficiently. But, in some cases the relaxing of the constraints lead to an idealization of the problem that is not realistic. Therefore, to reduce the computational overhead and keep the solution realistic, an suboptimal algorithm will be proposed with no relaxing in the constraints.

\section{Proposed Mimo Transmit Structures ASSIGNMENT - SUbOPTIMAL SOLUTION}

Health et. al. in [3] using the nearest neighbor union bound wrote the probability of vector symbol error as an upper bound

$$
\operatorname{Pr}(\operatorname{error} \mid \mathbf{H}) \leq M N_{e} Q\left(\sqrt{\mathrm{SNR}_{\min } \frac{d_{\min }^{2}}{2}}\right),
$$

where $d_{m i n}^{2}$ is the squared minimum distance, $\mathrm{SNR}_{m}$ in is the minimum SNR, $N_{e}$ is the average number of neighbors of the per-antenna constellation and $Q$ is the function that defines the area under the gaussian probability density function. Using this bound the authors calculate approximately the error probability of the MIMO structures designed to diversity and multiplexing using the minimum distance of the constellation as

$$
\begin{gathered}
\operatorname{Pr}_{D}(\text { error } \mid \mathbf{H}) \leq\left(2^{\log _{2} \mathcal{M}_{D}}-1\right) Q\left(\sqrt{\frac{E_{s}}{2 N_{0}} d_{m i n, D}^{2}(\mathbf{H})}\right) \\
\operatorname{Pr}_{M}(\text { error } \mid \mathbf{H}) \leq\left(\mathcal{M}_{M}-1\right) Q\left(\sqrt{\frac{E_{s}}{2 N_{0}} d_{\text {min }, M}^{2}(\mathbf{H})}\right),
\end{gathered}
$$

respectively, where $d_{\min , D}^{2}(\mathbf{H})$ and $d_{\min , M}^{2}(\mathbf{H})$ are the minimum distance of the diversity and multiplexing MIMO 


$$
\begin{gathered}
\operatorname{Pr}_{D}(\text { error } \mid \mathbf{H}) \leq\left(2^{\log _{2} \mathcal{M}_{D}}-1\right) Q\left(\sqrt{\frac{E_{s}}{2 N_{0}} \frac{(M-1) \lambda_{1}^{2}+\lambda_{M}^{2}}{N} d_{m i n, d}^{2}}\right) \\
\operatorname{Pr}_{M}(\text { error } \mid \mathbf{H}) \leq\left(\mathcal{M}_{M}-1\right) Q\left(\sqrt{\frac{E_{s}}{2 N_{0}} \frac{\lambda_{M}^{2}}{N} d_{m i n, m}^{2}}\right)
\end{gathered}
$$

structures constellation at the receiver. The cardinality of the modulation scheme to diversity and multiplexing is given by $\mathcal{M}_{D}$ and $\mathcal{M}_{M}$, respectively.

Using the Rayleigh-Ritz theorem [4] it follows that

$$
\lambda_{\min }^{2}(\mathbf{H}) \frac{d_{\min , m}^{2}}{M} \leq d_{\min , M}^{2}(\mathbf{H}) \leq \lambda_{\max }^{2}(\mathbf{H}) \frac{d_{\min , m}^{2}}{M},
$$

where $\lambda_{\min }^{2}$ and $\lambda_{\text {max }}^{2}$ are the minimum and maximal singular value of the channel matrix $\mathbf{H}$. Getting the lower bound we reach the approximation for multiplexing MIMO scheme like proposed by authors as

$$
d_{\min , M}^{2}(\mathbf{H}) \geq \frac{\lambda_{\min }^{2}}{M} d_{\min , m}^{2},
$$

where $d_{m i n, m}^{2}(\mathbf{H})$ is the corresponding minimum distance of the normalized unit energy constellation to MIMO multiplexing structures. To MIMO diversity structures we can use the following approximation to the minimum distance

$$
\begin{aligned}
d_{m i n, D}^{2}(\mathbf{H}) & \leq \frac{1}{M}\|\mathbf{H}\|_{F}^{2} d_{m i n, d}^{2} \\
& \leq \frac{(M-1) \lambda_{1}^{2}+\lambda_{M}^{2}}{N} d_{m i n, d}^{2}
\end{aligned}
$$

where $d_{\text {min,d }}^{2}(\mathbf{H})$ is the corresponding minimum distance of the normalized unit energy constellation to MIMO diversity structures.

Thus, replacing $d_{\min , D}^{2}$ and $d_{\min , M}^{2}$ we achieve (11) and (12) that are error probabilities of the MIMO structures designed to diversity and multiplexing relating the minimum distance of the constellation and the MIMO channel matrix, represented by $\lambda_{1}, \ldots, \lambda_{M}$ that are the singular value of the channel matrix $\mathbf{H}$ with $\lambda_{k} \leq \lambda_{1}, k=1, \ldots, M$.

In this section we propose a suboptimal strategy to assign MIMO transmit schemes providing a suboptimal solution to (3). The proposed algorithm is summarized in Algorithm 1 for a (4Tx-4Rx) MIMO system, but the extension to other cases is straightforward.

The proposed algorithm starts by assigning an OSTBC G2 (Alamouti's STBC) [5] to the first two transmit antennas. Then it checks if the QoS requirement is fulfilled. If the QoS is satisfied, then we may increase the data rate, transmitting uncoded streams in the other layers, as in the VBLAST approach. On the other hand, if QoS is not satisfied with the OSTBC assignment in the first step of the algorithm, we assign to the other two transmit antennas other OSTBC G2 providing more diversity trying to satisfy the QoS requirement. Following this strategy, we can choose between diversity and

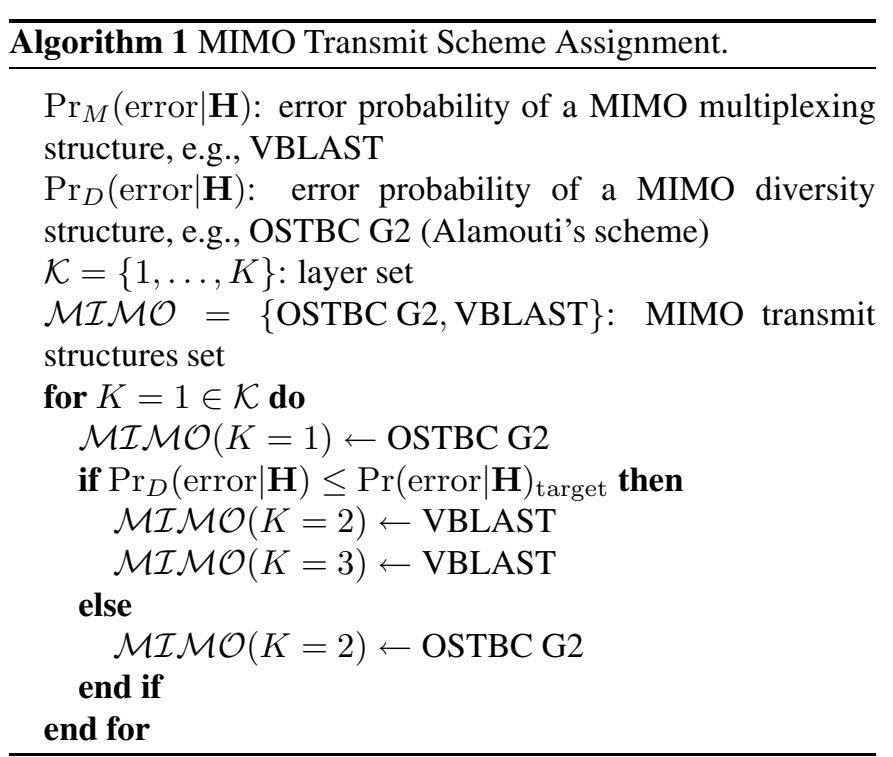

multiplexing online and respecting the QoS requirement. In other words, we are proposing a hybrid between the traditional diversity and multiplexing structures.

In general, the transmission process of these hybrid structures can be divided in layers, somewhat like VBLAST. However, in contrast to VBLAST, in the hybrid case a layer may consist of the stream of symbols at the output of a OSTBC, which is sent to a group of antennas, or of an uncoded stream, which is transmitted from a single antenna. Based on this concept of layers, hybrid structures combine pure diversity schemes (e.g. OSTBC G2) with pure spatial multiplexing schemes (e.g. VBLAST). In hybrid systems, some layers are space-time coded across two transmit antennas. For the remaining layers, a VBLAST approach is used if the QoS requirement is fulfilled. With this idea, hybrid MIMO structures achieve a compromise between spatial multiplexing and transmit diversity gains.

As shown in the Algorithm 1, if the first assignment of the OSTBC in the first two antennas is not enough to satisfy the QoS requirements, in the other two antennas other OSTBC G2 is assigned aiming to provide more diversity and achieve the QoS requirement. With this approach, we are assigning the first hybrid MIMO structure called G2+G2 described below.

\section{A. hybrid $G 2+G 2$}

The hybrid called G2+G2, is shown in Fig. 2(a). It employs a transmit antenna array with two vertical-layered OSTBC G2 space-time coding schemes, therefore, we have two OSTBC 


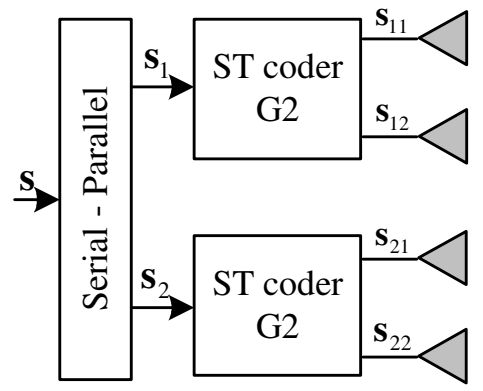

(a) Hybrid MIMO transmit structure G2+G2 with two STBC G2 and two multiplexing layers.

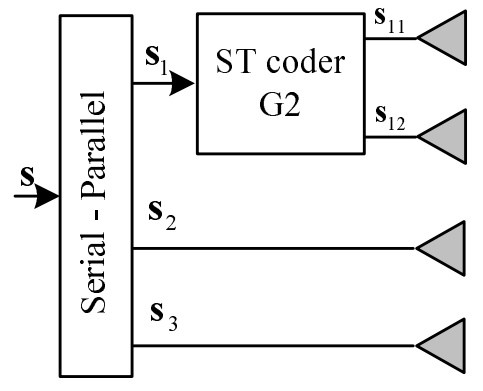

(b) Hybrid MIMO transmit structure $\mathrm{G} 2+1+1$ with one STBC G2 and three multiplexing layers.

Figure 2: Hybrid structures from the assignment algorithm.

layers in a parallel way then $K=2$ in this case. Observe that the four transmit antennas are divided into two space-time coding groups of two antennas each. The transmitted signals can be organized in an equivalent space-time coding matrix given by

$$
\mathbf{S}_{\mathrm{G} 2+\mathrm{G} 2}[T=1, T=2]=\left[\begin{array}{cccc}
s_{1} & s_{2} & s_{3} & s_{4} \\
-s_{2}^{*} & s_{1}^{*} & -s_{3}^{*} & s_{4}^{*}
\end{array}\right]^{T} .
$$

From (13), it can be seen that four information symbols (two from each multiplexing layer) are transmitted in two consecutive channel realizations. Thus, the effective data rate to this hybrid MIMO structure is $R_{G 2+G 2}=2$ symbols pcu.

On the other hand, if the first assigning is enough to QoS proposes, we could increase the data rate assigning VBLAST in the other antennas. This approach, came to the second hybrid MIMO structure called $\mathrm{G} 2+1+1$ as below.

\section{B. hybrid $G 2+1+1$}

The second hybrid is called $\mathrm{G} 2+1+1$ and is depicted in Fig. 2(b). As can be seen from the figure, this scheme consists of three spatial multiplexing layers $(K=3)$; the first layer is space-time coded using OSTBC G2, and the remaining are transmitted using VBLAST. The equivalent space-time coding matrix for the $\mathrm{G} 2+1+1$ scheme is given by

$$
\mathbf{S}_{\mathrm{G} 2+1+1}[T=1, T=2]=\left[\begin{array}{cccc}
s_{1} & s_{2} & s_{3} & s_{4} \\
-s_{2}^{*} & s_{1}^{*} & s_{5} & s_{6}
\end{array}\right]^{T} .
$$

In this hybrid structure, six information symbols (two from the first layer and four from the uncoded ones) are transmitted in two consecutive channel uses. Thus, the effective data rate to this hybrid MIMO structure is $R_{G 2+1+1}=3$ symbols pcu.

We should highlight that since the multiple layers see each other like interferer an interference cancellation algorithm like VBLAST one, e.g. successive interference cancelation (SIC), is mandatory in the receiver, such topic was regarded in [6].

In SIC, the layers are detected sequentially. Initially, the received signal in one symbol period generic $T$ (omitted here without loss of generality), $\mathbf{x}$ goes through a linear detector for layer 1 , whose output is used to produce a hard estimate of the symbols at this layer, $\hat{\mathbf{s}}_{1}$. Then, the contribution of layer 1 to the received signal is estimated and cancelled, generating the signal $x_{2}$. The process is then repeated. In general, at the $k$-th layer, the signal $\mathbf{x}_{k}$, hopefully free from the interference of layers $j<k$, goes through a linear detector that tries to mitigate the interference from layers $j>k$. A hard estimate of the symbol at this layer, $\hat{\mathbf{s}}_{k}$, is then produced, based on the output of this linear detector. Then, the contribution of this layer to the "received signal" $\mathbf{x}_{k}$ is estimated and cancelled. This procedure yields a modified received signal given by

$$
\mathbf{x}_{k+1}=\mathbf{x}_{k}-\hat{\mathbf{s}}_{k} \mathbf{h}_{k}
$$

where $\mathbf{h}_{k}$ is the $k$-th column of the matrix channel $\mathbf{H}$ corresponding to the channel gains associated to layer $k$, and $\hat{\mathbf{s}}_{k} \mathbf{h}_{i k}$ represents the estimated interference from the $k$-th layer. The result is that $\mathbf{x}_{k+1}$ is free from the interference coming from layers $1, \ldots, k$. This signal is then fed into the linear detector for the $(k+1)$-th layer. This technique is also known as nulling and cancelling algorithm. The performance of SIC can be further improved if the layers are detected in an appropriate order, resulting in ordered successive interference cancellation (OSIC). In this paper we will consider the SIC receiver for all the MIMO structures.

\section{Simulation Results}

In this section we present simulation results of our suboptimal assignment algorithm. The performance results are evaluated by means of numerical results from Monte-Carlo simulations. The curves are plotted against the average sum data rate per $\mathrm{Eb} / \mathrm{N}_{0}$ in $[\mathrm{dB}]$ for a given QoS (error probability error target) requirement. Unless otherwise noted, all schemes employ binary-phase shift-keying (BPSK) modulation.

The results presented in Figures 3 to 4 show the average sum data rate of the proposed algorithm in this paper for different QoS requirement for $10^{4}$ channel realizations. Here, the sum data rate $R_{\text {sum }}$ is the rate summed among all the layers as

$$
R_{\mathrm{sum}}=\sum_{k=1}^{K} R_{k}
$$




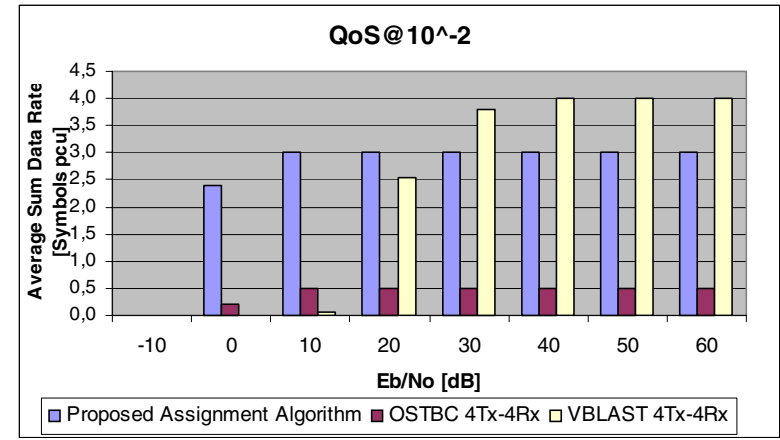

Figure 3: Average sum data rate for a QoS requirement of $10^{-2}$.

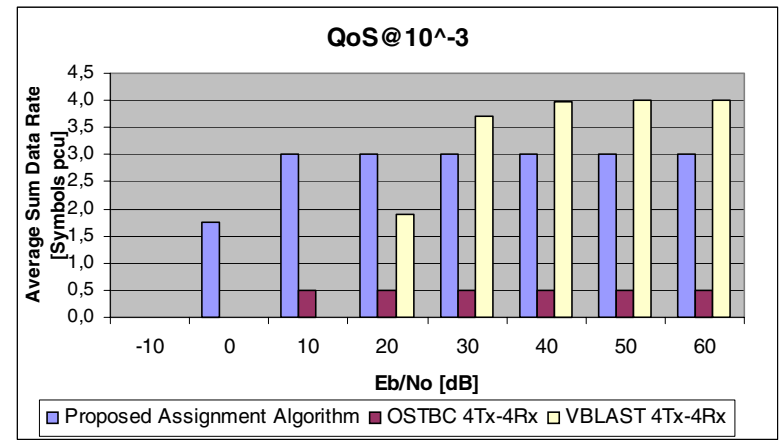

Figure 4: Average sum data rate for a QoS requirement of $10^{-3}$.

As benchmark using Equations (11) and (12), we also show the data rate achieved by schemes that just exploit diversity or multiplexing under the same QoS requirement. Further, we consider the fact that if the QoS is not fulfilled an outage occurs, thus, the data rate contribution is zero in this case.

From the results we can see that our approach is capable of adapting to the changes in the MIMO channel, achieving higher sum rates while respecting a defined QoS requirements for a intermediary range of $\mathrm{Eb} / \mathrm{N}_{0}$. When we increase the QoS requirements (e.g., error probability of $10^{-2}$ to $10^{-3}$ ), to pure OSTBC structure an outage occurs to an $\mathrm{Eb} / \mathrm{N}_{0}$ lower than $10 \mathrm{~dB}$ and in this case we are achieve the maximal data rate possible to our proposal, 3 symbols pcu. Comparing with the VBLAST, our proposal has a penalty of 1 symbol pcu to high $\mathrm{Eb} / \mathrm{N}_{0}$ values.

In summary, the OSTBC achieves the QoS requirement in parts of the $\mathrm{Eb} / \mathrm{N}_{0}$ range considered, but with lower average data rate. On the other hand, although VBLAST can achieve a sum data rate of 4 symbols pcu, it cannot respect the QoS requirement for the low and intermediary values of $\mathrm{Eb} / \mathrm{N}_{0}$. Therefore, our approach is feasible in capture the tradeoff present in the MIMO channel between diversity and multiplexing.

Figure 5 we compare our assignment approach based on the error probability to diversity and multiplexing in terms of the bit error rate $(\mathrm{BER})$ versus $\mathrm{Eb} / \mathrm{N}_{0}$ in $\mathrm{dB}$, here we assume as benchmark the VBLAST approach using SIC receiver. Let the

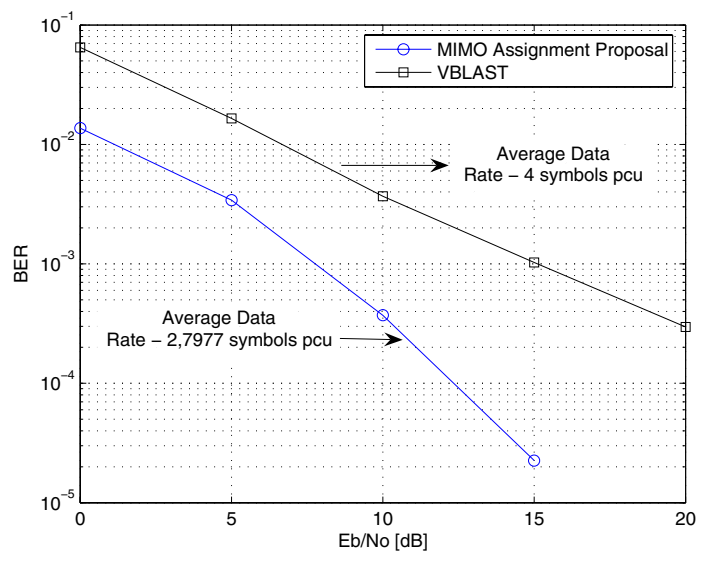

Figure 5: Bit error rate versus $\mathrm{Eb} / \mathrm{N}_{0}$ for a VBLAST (4Tx-4Rx) MIMO system and MIMO assignment proposal based on the error probability.

system be a (4Tx-4Rx) MIMO system. By the result we can see that our assignment approach provides a significant gain the BER performance adapting the MIMO transmit structure in accordance of the current MIMO channel using the error probability. Compared with the VBLAST scheme we can achieve a gain of $7 \mathrm{~dB}$ to a BER equals to $10^{-3}$ loosing just about 1.2 symbols pcu.

\section{CONCLUSIONS AND PERSPECTIVES}

In this paper, we present a suboptimal algorithm to solve the problem of MIMO transmit structure assignment in a MIMO wireless channel respecting a QoS requirement. Using our approach, we can achieve an increase in the average data rate compared with OSTBC and VBLAST approaches to low and intermediary values of $\mathrm{Eb} / \mathrm{N}_{0}$. As a perspective of this work is the impact of such proposal when regarding wit correlated MIMO channel models.

\section{REFERENCES}

[1] G. J. Foschini, "Layered Space-Time Architecture for Wireless Communications in a Fading Environment when using Multiple Antennas," Bell Labs Tech. J., v.1, n.2, pp.41-59, 1996.

[2] L. Zheng and D. Tse, "Diversity and Multiplexing: A Fundamental Tradeoff in Multiple Antenna Channels," IEEE Transactions on Information Theory, vol. 49, pp. 1073-96, May 2003.

[3] R. W. Heath Jr. and A. J. Paulraj, "Switching Between Diversity and Multiplexing in MIMO Systems," IEEE Transactions on Communications, vol. 53, no. 06, pp. 962-968, June 2005.

[4] Gene H. Golub, and Charles F. Van Loan, Matrix Computations, 3rd ed. Baltimore, MD: Johns Hopkins Univ. Press, 1996.

[5] S. Alamouti, "A simple transmit diversity technique for wireless communications," IEEE Journal of Selected Areas in Communications, vol.16, pp.1451-1458, Oct 1998.

[6] W. C. Freitas Jr., F. R. P. Cavalcanti, R. R. Lopes, "Hybrid Transceiver Schemes for Spatial Multiplexing and Diversity in MIMO Systems", Journal of Communication and Information Systems (JCIS), v. 20, no. 03, pp. 63-76, December 2005. 